\title{
Response to Richter and Vaezi
}

Gyawali, C. Prakash MD; Baker, Jason R. PhD²; Moshiree, Baha MD²; Rao, Satish MD; Neshatian, Leila

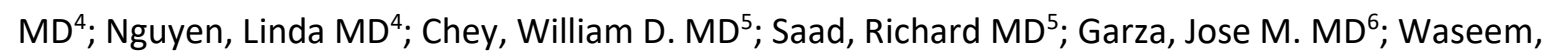
Shamaila MD; Khan, Abraham R. MD; Pandolfino, John E. MD ${ }^{9}$

${ }^{1}$ Washington University School of Medicine, St. Louis, Missouri, USA;

${ }^{2}$ Atrium Health, University of North Carolina, Charlotte, North Carolina, USA;

${ }^{3}$ Augusta University, Augusta, Georgia, USA;

${ }^{4}$ Stanford University, Stanford, California, USA;

${ }^{5}$ University of Michigan, Ann Arbor, Michigan, USA;

${ }^{6} \mathrm{GI}$ Care for Kids, Children's Healthcare of Atlanta, Atlanta, Georgia, USA;

${ }^{7}$ Indiana University, Indianapolis, Indiana, USA;

${ }^{8}$ New York University Langone Health, New York, New York, USA;

${ }^{9}$ Northwestern University, Chicago, Illinois, USA.

Correspondence: C. Prakash Gyawali, MD. E-mail: cprakash@wustl.edu.

We thank Richter and Vaezi $\left({ }^{1}\right)$ for their perspectives on our recent publication of the ANMS/ACG recommendations for successful reopening of motility laboratories in the coronavirus disease (COVID)19 era, and we are encouraged that our recommendations have proved helpful $\left({ }^{2}\right)$. Similar to the experiences of Richter and Vaezi, most of our motility centers are up to full capacity in performing motility studies. Among centers that have performed routine COVID-19 testing, our positivity rates have been similarly low $(0.2 \%-1.5 \%)$, but all positive cases were encountered in asymptomatic individuals. The pandemic continues to evolve, with varying community positivity rates around the country and varying local and institutional guidelines for testing and screening. Clearly, a one-size-fits-all approach is not going to be optimal.

We acknowledge that we are now learning more about the risk of transmission of the COVID-19 virus during endoscopy and motility laboratory procedures, and the risk seems to be low $\left({ }^{1,3}\right)$. Guidelines for endoscopy have evolved, and many gastrointestinal societies worldwide recommend careful screening for symptoms, contact, and temperature before confirmation of the endoscopy appointment in lieu of routine testing; some also recommend follow-up with the patient 14 days after the procedure $\left({ }^{4}\right)$. The approach outlined by Richter and Vaezi falls along similar concepts, and their experiences demonstrate that screening without routine testing has value as a successful approach to running a motility laboratory.

This is the author's manuscript of the work published in final form as:

Gyawali, C. P., Baker, J. R., Moshiree, B., Rao, S., Neshatian, L., Nguyen, L., Chey, W. D., Saad, R., Garza, J. M., Waseem, S., Khan, A. R., \& Pandolfino, J. E. (2021). Response to Richter and Vaezi. Official Journal of the American College of Gastroenterology, 116(1), 214-215. https://doi.org/10.14309/ajg.0000000000001021 
There are 3 factors that need additional consideration. First, routine symptom and contact screening over the telephone requires time and personnel, which could offset any cost savings from not performing COVID-19 tests. In most motility centers, motility personnel perform this additional screening, and one could make the argument that their time is better spent performing motility studies. Second, local and institutional guidelines have to be taken into consideration because some institutions continue to require testing for COVID-19 before invasive and endoscopic procedures $\left({ }^{5}\right)$. Finally, one has to account for the fact that inserting a nasal probe in an awake patient might result in sputtering, retching, sneezing, and, more importantly, coughing, all of which increase the risk of aerosolization $\left({ }^{6}\right)$. This can be of risk to the operator performing manometry and catheter-based reflux monitoring procedures, especially if the local prevalence of asymptomatic COVID-19 infections is high $\left({ }^{7}\right)$.

Currently, it might be reasonable to proceed with a symptom-and-contact screening approach, complemented with temperature checks, as long as the above-outlined factors are taken into consideration. If this approach is pursued without COVID-19 testing, we recommend use of full personal protective equipment, including gloves, face shield or goggles, and gown, during motility procedures with risk of aerosolization, particularly esophageal motility and reflux monitoring studies. As our understanding of the COVID-19 infection evolves, there will no doubt be further adjustments to published recommendations to enhance safety of both motility laboratory personnel and the patients they serve.

\section{CONFLICTS OF INTEREST}

Guarantor of the article: C. Prakash Gyawali, MD.

Specific author contributions: All authors contributed equally to the manuscript.

Financial support: No funding was obtained.

Potential competing interests: C.P.G.: consulting: Medtronic, Diversatek, Ironwood, Quintiles, Isothrive. J.R.B.: Consultant: Medtronic, Diversatek. B.M.: Research support: Medtronic. S.R.: None. L. Ne: None; L. Ng: None; W.D.C.: consulting: Commonwealth Diagnostic International, Gemelli, Progenity; grant funding: Commonwealth Diagnostics International. R.S.: None. J.M.G.: consulting: Medtronic; speaking: Abbott, Biogaia, Medtronic. S.W.: None. A.R.K.: consulting: Medtronic, Progenity. J.E.P.: consulting: Medtronic, Diversatek; speaking: Medtronic, Diversatek, Ethicon; stock options: Crospon; licensing arrangement: Medtronic. 


\section{REFERENCES}

1. Richter J, Vaezi M. Letter to the Editor-An alternative recommendation by experienced esophagologists. Am J Gastroenterol 2021;116(1):214.

2. Baker JR, Moshiree B, Rao S, et al. American Neurogastroenterology and Motility Society Task Force recommendations for resumption of motility laboratory operations during the COVID-19 pandemic. Am J Gastroenterol 2020;115(10):1575-83.

3. Elli L, Tontini GE, Filippi E, et al. Efficacy of endoscopic triage during the Covid-19 outbreak and infective risk. Eur J Gastroenterol Hepatol 2020;32:1301-4.

4. Castro Filho EC, Castro R, Fernandes FF, et al. Gastrointestinal endoscopy during the COVID-19 pandemic: An updated review of guidelines and statements from international and national societies. Gastrointest Endosc 2020;92:440-5.e6.

5. Peery AF, Arora S, Shaheen NJ. Reviving routine gastrointestinal endoscopy in the COVID-19 era. Am J Gastroenterol 2020;115:1376-9.

6. Workman AD, Welling DB, Carter BS, et al. Endonasal instrumentation and aerosolization risk in the era of COVID-19: Simulation, literature review, and proposed mitigation strategies. Int Forum Allergy Rhinol 2020;10:798-805.

7. Baker JR, Moshiree B, Pandolfino J, et al. Response to the Letter: How do we reopen our motility laboratory safely and efficiently? Neurogastroenterol Motil 2020;32:e13969. 